\title{
Tanshinone IIA promotes cardiac differentiation and improves cell motility by modulating the Wnt/ $\beta$-catenin signaling pathway
}

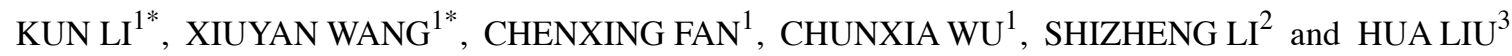 \\ Departments of ${ }^{1}$ Clinical Laboratory and ${ }^{2}$ General Surgery, The First Affiliated Hospital of Jinzhou Medical University; \\ ${ }^{3}$ Institute of Eyes, Jinzhou Medical University, Jinzhou, Liaoning 121001, P.R. China
}

Received December 2, 2019; Accepted June 1, 2020

DOI: $10.3892 / \mathrm{mmr} .2020 .11272$

\begin{abstract}
Although the cardiovascular pharmacological actions of Tanshinone IIA (TanIIA) have been extensively studied, research on its roles in cardiac regeneration is still insufficient. The present study employed the cardiac myoblast cell line H9c2 to evaluate the possible roles of TanIIA in cardiac regeneration. It was found that certain concentration of TanIIA inhibited cell proliferation by suppressing the expression of proteins related to the cell cycle [cyclin dependent kinase (CDK)4, CDK6 and cyclin D1] and proliferation [c-Myc, octamer-binding transcription factor 4 (Oct4) and proliferating cell nuclear antigen (PCNA)] without inducing apoptosis. In this process, the expression of cardiac troponin in the treated cells was significantly increased and the migration of the treated cells toward the wound area was significantly enhanced. Meanwhile, TanIIA inhibited the canonical signaling pathway through increasing the expression of glycogen synthase kinase $3 \beta$ (GSK-3 $\beta$ ) and adenomatous polyposis coli (APC) and increased the expression of Wnt11 and Wnt5a in the noncanonical Wnt signaling pathway. Following $\beta$-catenin agonist WAY-262611 intervention, the effect of TanIIA on the promotion of cardiac differentiation and improved cell migration was significantly reduced. In conclusion, it was hypothesized that TanIIA could promote cardiac differentiation and improve cell
\end{abstract}

Correspondence to: Dr Shizheng Li, Department of General Surgery, The First Affiliated Hospital of Jinzhou Medical University, 2 Section 5 Renmin Street, Guta, Jinzhou, Liaoning 121001, P.R. China

E-mail: 1szlklym@163.com

Dr Hua Liu, Institute of Eyes, Jinzhou Medical University, 40 Section 3 Songpo Road, Linghe, Jinzhou, Liaoning 121001, P.R. China E-mail: 1h50515@163.com

*Contributed equally

Abbreviations: TanIIA, Tanshinone IIA; SM, Salvia miltiorrhiza Bunge; DMEM, Dulbecco's modified Eagle's medium; HRP, horseradish peroxidase

Key words: Tanshinone IIA, H9c2 cells, cardiac differentiation, cell motility, Wnt/ $\beta$-catenin signaling motility by modulating the Wnt/ $\beta$-catenin signaling pathway. These results suggest that TanIIA may play beneficial roles in myocardial regeneration following stem cell transplantation.

\section{Introduction}

Ischemic heart disease is a serious threat to human health (1). Although treatments such as thrombolysis, interventional therapy and coronary artery bypass graft are effective in improving myocardial ischemia in patients, these treatments cannot make up the loss of cardiomyocytes at myocardial ischemia, thereby affecting the cardiac functions of patients, even eventually causing heart failure for some patients (2). With improved understanding of stem cells, the use of their differentiation potential to regenerate cardiomyocytes has become a new strategy for the treatment of ischemic heart disease $(2,3)$. Since there are recurrent issues associated with the direct transplantation of stem cells including low engraftment rate, arrhythmia and tumorigenesis (4-6), it is generally believed that pre-differentiation of stem cells into cardiac precursor cells for transplantation is safer and more effective than direct transplantation of stem cells $(7,8)$. Therefore, improving the efficiency of pre-differentiated cardiac precursor cells to further differentiate into cardiomyocytes and promote the migration of these cells to the injured area has become a focus of research.

Tanshinone IIA (TanIIA) is a key active component of Salvia miltiorrhiza Bunge (SM), a deciduous perennial plant native to China; it has been demonstrated to prevent the incidence of ischemic heart disease by lowering blood lipids (9), alleviating atherosclerosis (10) and preventing thrombosis (11). It improves the tissue environment of damaged myocardium by inhibiting inflammatory responses (12), dilating coronary arteries (13), increasing coronary blood flow (14) and reducing myocardial hypoxia $(13,14)$. In addition, TanIIA can suppress the ischemia-induced arrhythmia and reduce myocardial infarct size $(15,16)$. Our previous study demonstrated that TanIIA could induce human placenta-derived mesenchymal stem cells to differentiate into cardiomyocytes (17). In view of the above, it was hypothesized that further TanIIA treatment following cell transplantation may promote the cardiac regeneration efficiency of transplanted cells.

Wnt signaling plays an important role in heart development and particularly in cardiomyocyte differentiation, and the canonical and the noncanonical Wnt pathways are involved 
during various stages of cardiac differentiation (18). It is well known that the heartdevelops from the mesoderm(19).Following mesoderm formation, inhibition of canonical Wnt/ $\beta$-catenin signaling can promote cardiac differentiation (19-21), while noncanonical Wnt signaling can inhibit canonical Wnt/ $\beta$-catenin signaling through multiple mechanisms, thereby further promoting cardiac differentiation $(22,23)$. In addition to its role in heart development and cardiomyocyte differentiation, Wnt/ $\beta$-catenin signaling is also involved in the regulation of cell migration (24). Therefore, it was hypothesized that TanIIA may promote the differentiation of pre-differentiated cardiac precursor cells into cardiomyocytes and improve the motility of these cells to the injured area by modulating the $\mathrm{Wnt} / \beta$-catenin signaling pathway. $\mathrm{H} 9 \mathrm{c} 2$, a permanent cardiac cell line isolated from embryonic rat heart, is used as an in vitro cell model for cardiac differentiation due to its ability to differentiate into cardiomyocytes $(25,26)$. Since the application of human-derived cardiac progenitor stem cells is restricted by some factors, such as the supply and ethics (27), the present study employed $\mathrm{H} 9 \mathrm{c} 2$ cells to simulate the pre-differentiated cardiac precursor cells to corroborate our aforementioned speculation in vitro.

\section{Materials and methods}

Cell culture. H9c2 cells were cultured in Dulbecco's modified Eagle's medium (DMEM) supplemented with $10 \%$ fetal bovine serum and $1 \%$ penicillin-streptomycin in $5 \% \mathrm{CO}_{2}$ at $37^{\circ} \mathrm{C}$. In all experiments, cells in logarithmic phase were used.

Evaluation of the effect of TanIIA on the proliferation of $\mathrm{H} 9 \mathrm{c} 2$ cells using MTS assay. H9c2 cells were inoculated in 96-well plates at an initial density of 5,000 cells/well and cultured in DMEM supplemented with $10 \%$ fetal bovine serum and $1 \%$ penicillin-streptomycin. The next day, cells were treated with TanIIA at different concentrations $(0.01,0.02,0.04,0.06,0.08$, $0.1,0.2,0.4,0.6,0.8,1,5,10$ and $20 \mathrm{mg} / \mathrm{l}$ ) for 4 days. At the end of the incubation, cell proliferation was determined using 3-(4,5-diethylthiazol-2-yl)-5-(3-carboxymethoxyphenyl)-2-(4sulfophenyl)-2H-tetrazolium (MTS) assay following the manufacturer's protocols (Promega Corporation). Over the course of the experiment, the culture medium was not changed for 5 days from the beginning of cell seeding to the next day of dosing until the final detection, to avoid the detected cells being washed away, and the influence of changing culture medium on the experimental result. After 4 days of incubation with or without TanIIA, cell proliferative activity was also evaluated by the expression changes of proteins related to cell cycle [cyclin dependent kinase (CDK)4, CDK6 and cyclin D1] and proliferation [c-Myc, octamer-binding transcription factor 4 (Oct4) and proliferating cell nuclear antigen (PCNA)] Meanwhile, cell apoptosis was evaluated by the expression changes in caspase- 3 and cleaved caspase- 3 . Changes in the expression levels of the aforementioned proteins were evaluated by western blotting.

Evaluation of the effect of TanIIA on the expression of cardiac troponin (cTn) using immunohistochemistry. H9c2 cells were treated with TanIIA or TanIIA combined with $\beta$-catenin agonist WAY-262611 for 7 days. The treated cells were washed with
PBS and fixed with $4 \%$ paraformaldehyde for $20 \mathrm{~min}$ at room temperature, permeabilized with $0.2 \%$ Triton X-100 for $10 \mathrm{~min}$ at room temperature, and blocked with a serum blocking solution (cat. no. P0260; Beyotime Institute of Biotechnology) for $5 \mathrm{~min}$ at room temperature. The cells were then incubated with cTnI (1:100 dilution; cat. no. ab47003; Abcam) and cTnT (1:100 dilution; cat. no. ab115134; Abcam) overnight at $4^{\circ} \mathrm{C}$, followed by incubation with horseradish peroxidase (HRP) conjugated goat anti-rabbit IgG (1:5,000 dilution; cat. no. ab6721; Abcam) for $10 \mathrm{~min}$ at room temperature. Following an incubation period of $10 \mathrm{~min}$ with streptavidin-HRP and $5 \mathrm{~min}$ with DAB (cat. no. P0203; Beyotime Institute of Biotechnology), cells were observed under a microscope (magnification, x200) (Leica dMI1; Leica Microsystems, Inc.). For each step, cells were washed three times with PBS.

Evaluation of the effect of TanIIA on cell motility using scratch assay. H9c2 cells were treated with TanIIA or TanIIA combined with $\beta$-catenin agonist WAY-262611 for 7 days, and then harvested. The cells were replated in 6-well plates and cultured to full confluence. The cell layers were scratched with a $200-\mu 1$ pipette tip, and then washed thoroughly and further incubated in serum-free medium for $48 \mathrm{~h}$. Images were captured at 0 and $48 \mathrm{~h}$ using an inverted microscope (magnification, x200) (Leica dMI1; Leica Microsystems, Inc.). In our experiments, wound closure $=\left(\right.$ wound area of $\mathrm{T}_{0}$-wound area of $\mathrm{T}_{\mathrm{t}}$ )/wound area of $\mathrm{T}_{0} \times 100 \%$.

Evaluation of the effect of TanIIA on the expression of cTn and the key components in the canonical and noncanonical Wnt pathway using western blotting. H9c2 cells were treated with TanIIA for 4 days or treated with TanIIA or TanIIA combined with $\beta$-catenin agonist WAY-262611 for 7 days. The treated cells were washed with pre-cooled PBS twice and then collected. Total proteins were extracted with cell lysis buffer (cat. no. P0013b; Beyotime Institute of Biotechnology) according to the manufacturer's protocol. The protein quantity was determined by bicinchoninic acid assay (BioTek Instruments, Inc.). Equal quantities of protein (30 $\mu \mathrm{g})$ were respectively separated with sodium dodecyl sulfate-polyacrylamide gel electrophoresis on 8 or $10 \%$ gel and then transferred onto polyvinylidene difluoride membranes. The membranes were blocked with 5\% non-fat milk for $1 \mathrm{~h}$ at room temperature. Membranes containing protein extracted from cells treated for 4 days were incubated overnight at $4^{\circ} \mathrm{C}$ with the following antibodies: GAPDH (1:1,000 dilution; cat. no. ab9485), $\beta$-actin (1:1,000 dilution; cat. no. ab8227), CDK4 (1:500 dilution; cat. no. WL02274), CDK6 (1:500 dilution; cat. no. WL03460), Cyclin D1 (1:500 dilution; cat. no. WL01435a), c-Myc (1:500 dilution; cat. no. WL01781), octamer-binding transcription factor 4 (Oct4; 1:500 dilution; cat. no. WL02020), PCNA (1:500 dilution; cat. no. WL02208), caspase-3 (1:500 dilution; cat. no. WL04004), or cleaved caspase-3 (1:500 dilution; cat. no. WL01992; GAPDH and $\beta$-actin from Abcam, others from Wanleibio Co., Ltd.), and the membranes containing protein extracted from cells treated for 7 days were incubated overnight at $4^{\circ} \mathrm{C}$ with the following antibodies: GAPDH, $\beta$-actin, CDK4, CDK6, cyclin D1, c-Myc, Oct4, PCNA, glycogen synthase kinase 3 (GSK-3 $\beta$; 1:1,000 dilution; cat. no. ab131356; Abcam), adenomatous 
polyposis coli (APC; 1:1,000 dilution; cat. no. ab40778; Abcam), $\beta$-catenin (1:1,000 dilution; cat. no. ab2365; Abcam), Wnt 5a (1:1,000 dilution; cat. no. ab174963; Abcam), Wnt 11 (1:1,000 dilution; cat. no. ab31962; Abcam), cTnI (1:1,000 dilution; cat. no. ab47003; Abcam), cTnT (1:1,000 dilution; cat. no. ab115134; Abcam), C-X-C chemokine receptor type 4 (CXCR4; 1:500 dilution; cat. no. WL02421; Wanleibio Co., Ltd.), or C-C chemokine receptor type 2 (CCR2; 1:500 dilution; cat. no. WL03663; Wanleibio Co., Ltd.). This was followed by incubation with HRP-conjugated goat anti-rabbit IgG (1:5,000 dilution; cat. no. ab6721; Abcam) for $2 \mathrm{~h}$ at room temperature. Antibodies were detected using the chemiluminescence detection kit (Omega Lum G; Aplegen Inc.), and the detection results were quantified by ImageJ (version 1.4.3.67; National Institutes of Health).

Statistical analysis. Each experiment was repeated at least three times. All data were presented as mean \pm standard deviation. Statistical analysis was performed using SPSS 13.0 software (SPSS, Inc). A one-way analysis of variance was used for comparisons among the groups, and Bonferroni's post hoc test was used to compare the means of each two groups. $\mathrm{P}<0.05$ was considered to indicate a statistically significant difference.

\section{Results}

TanIIA inhibits cell proliferation of $\mathrm{H} 9 \mathrm{c} 2$ cells. In order to select a reasonable concentration of TanIIA for the present study, the proliferation of $\mathrm{H} 9 \mathrm{c} 2$ cells treated with TanIIA in a concentration range from 0 to $20 \mathrm{mg} / \mathrm{l}$ for 4 days was evaluated using MTS assay. Starting at $0.4 \mathrm{mg} / 1$, TanIIA induced a marked reduction in cell number, which reached a plateau at $0.6 \mathrm{mg} / 1$ which ended at $2 \mathrm{mg} / \mathrm{l}$; there was no significant difference at $2 \mathrm{mg} / \mathrm{l}$ compared with $0.6 \mathrm{mg} / \mathrm{l}$ (Fig. 1A). Based on this result, $0.6 \mathrm{mg} / \mathrm{l}$ was selected as the concentration used throughout the present study to evaluate the effect of TanIIA on cardiac differentiation.

The inhibition of cell proliferation treated with $0.6 \mathrm{mg} / \mathrm{l}$ TanIIA for 4 days was also demonstrated by the decreased expression of proteins related to the cell cycle (CDK4, CDK6 and cyclin D1) (Fig. 1B-a) and proliferation (c-Myc, Oct4 and PCNA) (Fig. 1B-b) when compared to the control. To further corroborate the inhibition of cell proliferation was not caused by apoptosis, cell apoptosis and the expression of caspase- 3 and cleaved caspase- 3 were evaluated. The results demonstrated that cell proliferation inhibition induced by TanIIA was not accompanied by changes in cell apoptosis or expression of caspase-3 and cleaved caspase-3 (Fig. 1B-c).

TanIIA promotes the expression of cTn. Immunohistochemistry was used to evaluate the expression of cTn. The results demonstrated that the expression of cTnI and cTnT was increased following TanIIA incubation for 7 days. Meanwhile, some cells were markedly increased in size, indicating that cardiomyotubes area was enhanced (Fig. 2A). This suggested that TanIIA promoted cardiac differentiation of $\mathrm{H} 9 \mathrm{c} 2$ cells. In addition, the aforementioned results were further corroborated by the significantly increased expression of cTnI and cTnT in the cells treated with $0.6 \mathrm{mg} / 1$ TanIIA for 7 days as evaluated by western blot analysis (Fig. 2B).

TanIIA modulates the expression of key components of the canonical and noncanonical Wnt signaling pathway. Western blotting was used to evaluate the expression of key components of the canonical and noncanonical Wnt signaling pathway. The results indicated that following incubation of H9c2 cells with $0.6 \mathrm{mg} / 1$ TanIIA for 7 days, the expression of the negative regulator GSK-3 $\beta$ and APC of the canonical Wnt signaling pathway was increased, as expected, and the expression of the core protein $\beta$-catenin of the canonical Wnt signaling pathway was significantly decreased (Fig. 3A). The expression of two non-canonical Wnt signaling members Wnt5a and Wnt11 was significantly increased (Fig. 3B).

TanIIA increases cell motility. Scratch assay was performed to evaluate the effect of TanIIA on the motility of treated cells. The results demonstrated that compared with those in the control group, a greater number of TanIIA-treated cells were migrated from the wound edge towards the wound area (Fig. 4A). Since CXCR4 and CCR2 are important chemokine receptors that regulates cell motility $(28,29)$, their expression in TanIIA-treated cells were next evaluated. The expression of CXCR4 and CCR2 in the cells treated with TanIIA was significantly increased (Fig. 4B).

$\beta$-catenin agonist WAY-262611 intervention results in upregulated expression of proteins related to cell cycle and proliferation in $\mathrm{H} 9 \mathrm{c} 2$ cells treated with TanIIA. Previous studies have demonstrated that following mesoderm formation, inhibition of canonical $\mathrm{Wnt} / \beta$-catenin signaling can promote cardiac differentiation $(19,20)$, while noncanonical Wnt signaling can inhibit canonical Wnt/ $\beta$-catenin signaling through multiple mechanisms, thereby further promoting cardiac differentiation $(22,23)$. The above results indicated that TanIIA may promote cardiac differentiation and improve cell motility by modulating canonical Wnt/ $\beta$-catenin signaling pathway. To further confirm the hypothesis, the expression changes of proteins related to cell cycle (CDK4, CDK6 and cyclin D1) and proliferation (c-Myc, Oct4 and PCNA) were assessed in TanIIA-treated cells following $\beta$-catenin agonist WAY-262611 intervention. The results suggested that following the intervention of $\beta$-catenin agonist WAY-262611, the expression of $\beta$-catenin (Fig. 5A) and proteins related to cell cycle (CDK4, CDK6 and cyclin D1) (Fig. 5B) or proliferation (c-Myc, Oct4 and PCNA) (Fig. 5C) in the TanIIA-treated $\mathrm{H} 9 \mathrm{c} 2$ cells were increased significantly, suggesting that the proliferative activity of the TanIIA-treated H9c2 cells was enhanced and might be accompanied by a reduction in cardiac differentiation.

$\beta$-catenin agonist WAY-262611 intervention results in decreased cTn expression and cell motility in TanIIA-treated cells. To confirm whether $\beta$-catenin agonist WAY-262611 intervention results in the increase in cell proliferation and the decrease in cardiac differentiation in TanIIA-treated cells, the expression of cTnI and cTnT was evaluated by immunohistochemistry. The results demonstrated that 


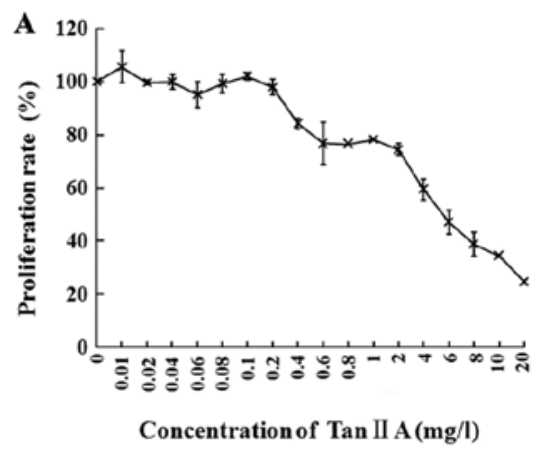

B a

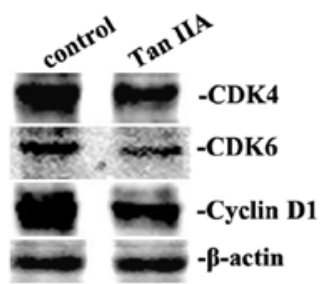

b

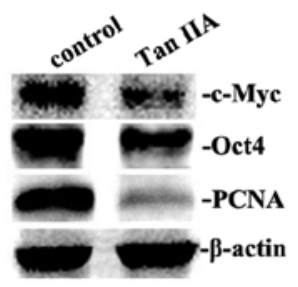

c

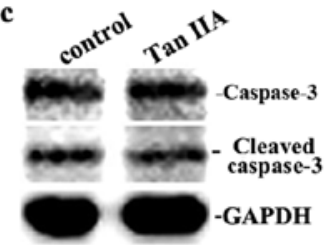

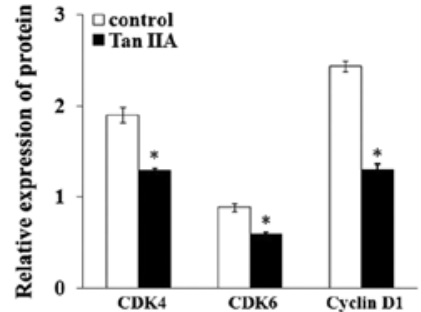
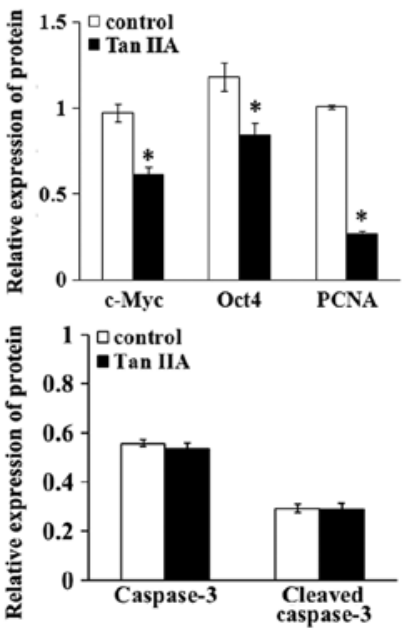

Figure 1. Effect of TanIIA on the proliferation of H9c2 cells. (A) H9c2 cells were incubated with increasing concentrations of TanIIA (0-20 mg/l) for 4 days and, at the end of the incubation, cell proliferation was determined using MTS assay. (B) H9c2 cells were incubated with or without $0.6 \mathrm{mg} / 1$ TanIIA for 4 days and, at the end of incubation, the expression changes in (a) CDK4, CDK6, cyclin D1, (b) c-Myc, Oct4, PCNA and (c) caspase-3 and cleaved caspase-3 were evaluated by western blotting. "P<0.05, significantly different from control. TanIIA, Tanshinone II A; CDK, cyclin dependent kinase; Oct 4 , octamer-binding transcription factor 4; PCNA, proliferating cell nuclear antigen.
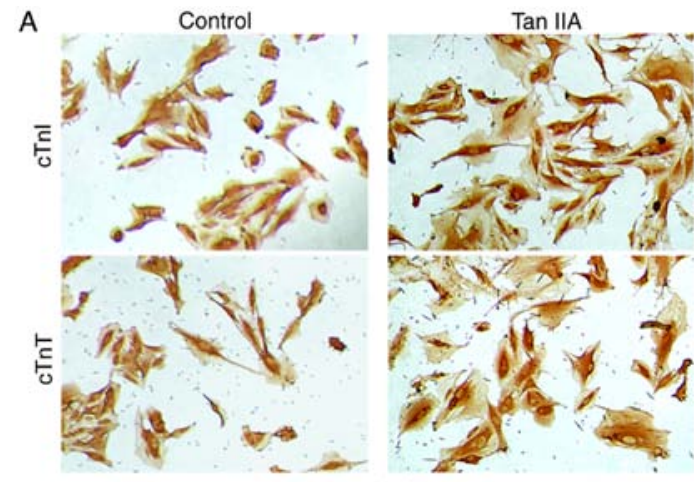

B
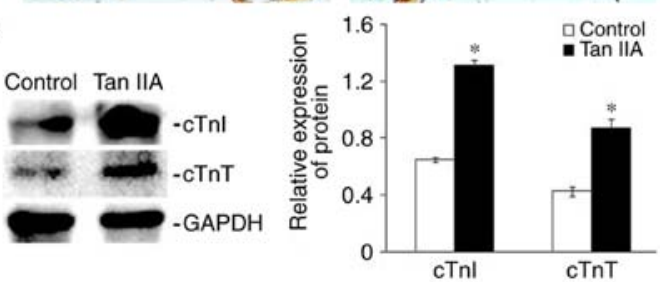

Figure 2. TanIIA promotes the expression of cTn. H9c2 cells were incubated with or without $0.6 \mathrm{mg} / \mathrm{l}$ TanIIA for 7 days. At the end of incubation, (A) immunohistochemistry was used to evaluate the expression of cTnI and $\mathrm{cTnT}$ (magnification, x200) and (B) western blotting was used to further corroborate expression of $\mathrm{cTnI}$ and $\mathrm{cTnT}$ in the cells. In the immunohistochemical study, cell staining intensity was found to be positively correlated with the expression level of cTn. ${ }^{*} \mathrm{P}<0.05$ vs. the control. TanIIA, Tanshinone II A; cTn, cardiac troponin.

compared with treatment with TanIIA alone, the expression of cTnI and cTnT in cells treated with TanIIA combined with WAY-262611 was markedly decreased (Fig. 6A), which was
A
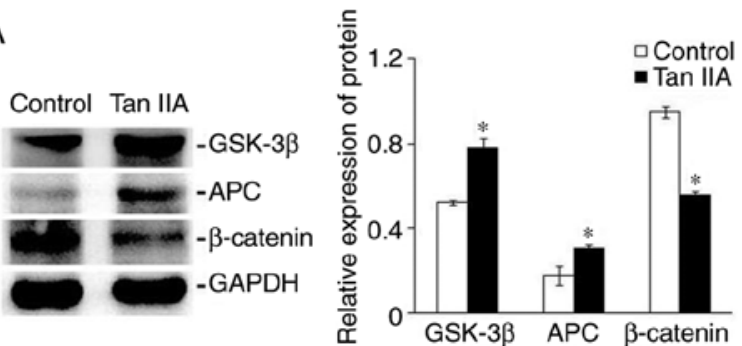

B
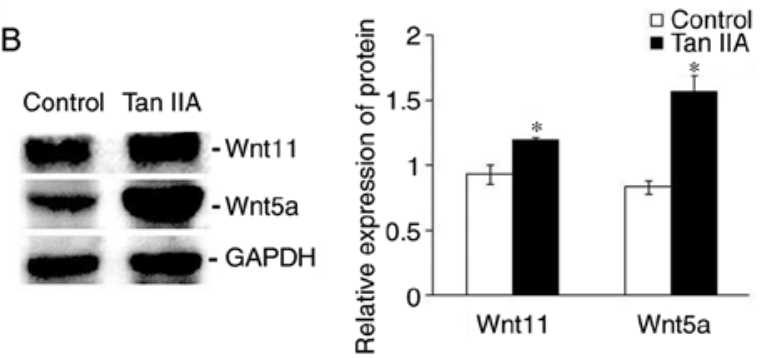

Figure 3. Effect of TanIIA on the expression of key components of the canonical and noncanonical Wnt signaling pathway. H9c2 cells were incubated with or without $0.6 \mathrm{mg} / 1$ TanIIA for 7 days. At the end of incubation, the expression of key components of the canonical and noncanonical Wnt signaling pathway was evaluated by western blotting. (A) Expression of GSK- $3 \beta$, APC and $\beta$-catenin. (B) Expression of Wnt $5 \mathrm{a}$ and Wnt11. ${ }^{*} \mathrm{P}<0.05$ vs. the control. TanIIA, Tanshinone II A; GSK-3 $\beta$, glycogen synthase kinase 3; APC, adenomatous polyposis coli.

further corroborated by western blot analysis (Fig. 6B). In addition, the motility of cells treated with TanIIA combined with WAY-262611 was decreased compared with treatment 


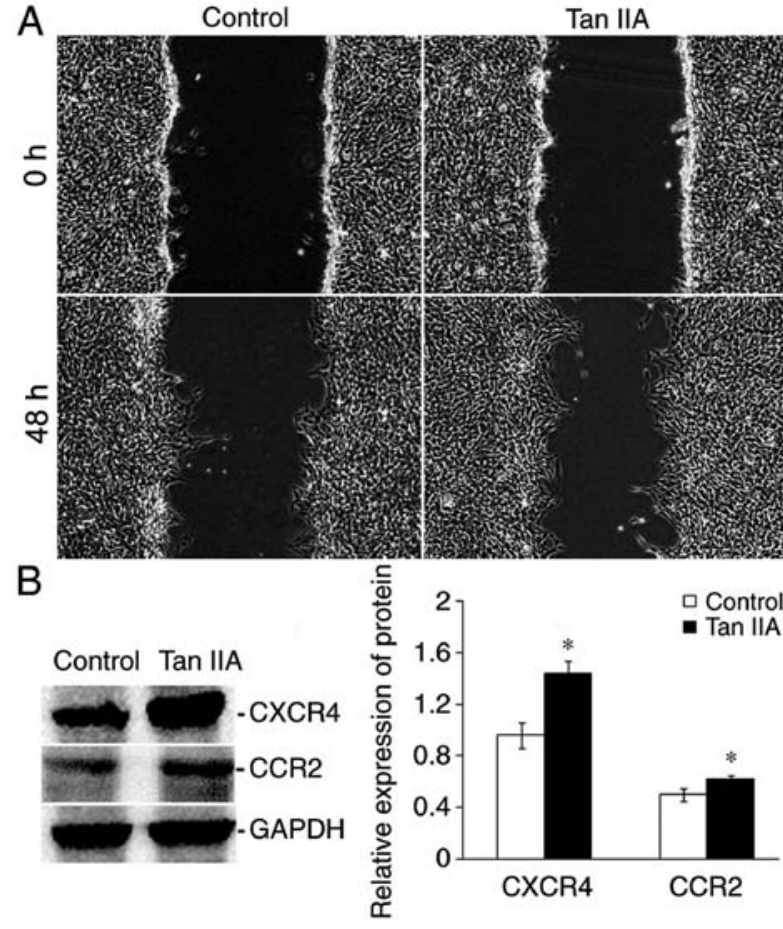

Figure 4. TanIIA enhances cell motility. H9c2 cells were incubated with or without $0.6 \mathrm{mg} / 1$ TanIIA for 7 days. At the end of incubation, (A) scratch assay was used to evaluate cell motility (magnification, $x 100$ ) and (B) western blotting was used to assess the expression of chemokine receptors related to cell motility CXCR 4 and CCR2. ${ }^{*} \mathrm{P}<0.05$ vs. the control. TanIIA, Tanshinone II A; CXCR4, C-X-C chemokine receptor type 4; CCR2, C-C chemokine receptor type 2 .

with TanIIA alone (Fig. 6C), and the expression of CXCR4 and CCR2 was also significantly decreased (Fig. 6D).

\section{Discussion}

Using the differentiation potential of stem cells to regenerate cardiomyocytes for treatment of ischemic heart disease has been studied for decades, and at present it is generally believed that pre-differentiation of stem cells into cardiac precursor cells for transplantation is safer and more effective than direct transplantation of stem cells $(7,8)$. Even so, previous preclinical studies $(8,30)$ have shown some limitations in the application of cardiac regeneration and the challenge of complete cardiac regeneration will require: i) Improvement of the recipient myocardial tissue environment before the implantation of cells, ii) reduction of the transplantation-mediated arrhythmia and iii) increase of the efficiency of the pre-differentiated cardiac precursor cells to further differentiate to functional cardiomyocytes and promotion of the migration of these cells to the injured area. Tanshinone IIA (TanIIA) is a key active component of Salvia miltiorrhiza Bunge and is widely used in China and other neighboring countries to prevent and treat cardiovascular disorders (15). Previous studies have reported that TanIIA can improve the tissue environment of damaged myocardium and suppress the ischemia-induced arrhythmia (12-14,31). Therefore, TanIIA may help solve the above problems i) and ii) facing cardiac regeneration. In addition, previous studies have also demonstrated that TanIIA can reduce myocardial
$\mathbf{A}$
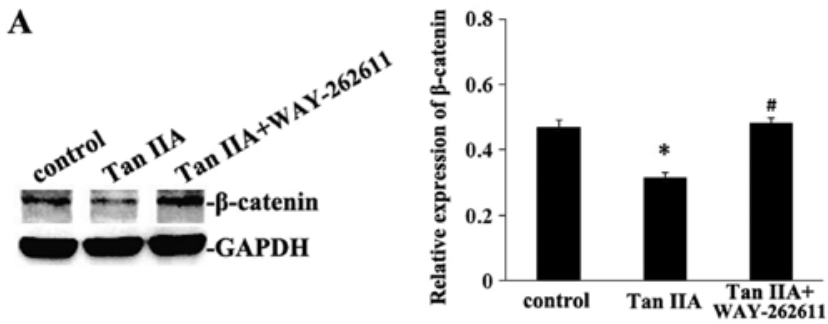

B
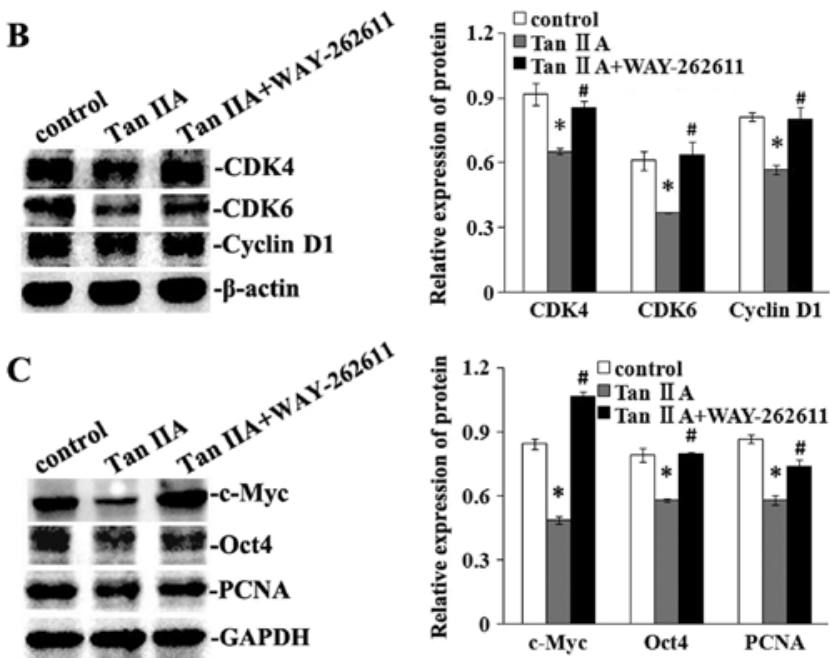

Figure 5. $\beta$-catenin agonist WAY-262611 intervention upregulates the expression of proteins related to cell cycle and proliferation in H9c2 cells treated with TanIIA. H9c2 cells were treated with TanIIA or TanIIA combined with $\beta$-catenin agonist WAY-262611 for 7 days. At the end of incubation, the expression changes of (A) $\beta$-catenin, (B) CDK4, CDK6, cyclin D1, and (C) c-Myc, Oct4 and PCNA were evaluated by western blotting. " $\mathrm{P}<0.05$ vs. the control; ${ }^{*} \mathrm{P}<0.05$ : vs. TanIIA alone. TanIIA, Tanshinone II A; CDK, cyclin dependent kinase; Oct4, octamer-binding transcription factor 4; PCNA, proliferating cell nuclear antigen.

infarct size $(16,32,33)$, and the results of the present study indicated that TanIIA could induce the differentiation of stem cells into cardiomyocytes $(17,34)$. Therefore, it was hypothesized that TanIIA also improves the efficiency of the pre-differentiated cardiac precursor cells to further differentiate into cardiomyocytes and promote the migration of these cells to the injured area. The present study employed $\mathrm{H} 9 \mathrm{c} 2$ cells to simulate the pre-differentiated cardiac precursor cells to corroborate the hypothesis. Since H9c2 cells have a certain proliferative capacity, and cardiomyocytes do not, when H9c2 cells are induced to differentiate into cardiomyocytes, cell numbers in the induction and differentiation group will be lower compared with the untreated group. Hence, here is a negative relationship between the proliferation and differentiation of H9c2 cells $(25,26)$. Therefore, the effect of TanIIA on the proliferation of $\mathrm{H} 9 \mathrm{c} 2$ cells was first evaluated. The results demonstrated that starting at $0.4 \mathrm{mg} / \mathrm{l}$, TanIIA induced a significant reduction in cell numbers, which reached a plateau at $0.6 \mathrm{mg} / \mathrm{l}$ and ended at $2 \mathrm{mg} / 1$, and there was no significant difference at $2 \mathrm{mg} / \mathrm{l}$ compared with $0.6 \mathrm{mg} / \mathrm{l}$. Meanwhile, dead cells were not observed in the culture medium. However, although TanIIA at a concentration higher than $2 \mathrm{mg} / \mathrm{l}$ could better inhibit the proliferation of $\mathrm{H} 9 \mathrm{c} 2$ cells, a large number of dead cells were observed in the culture medium, and the number of dead cells was positively correlated with the concentration of TanIIA, suggesting that the inhibition of cell 

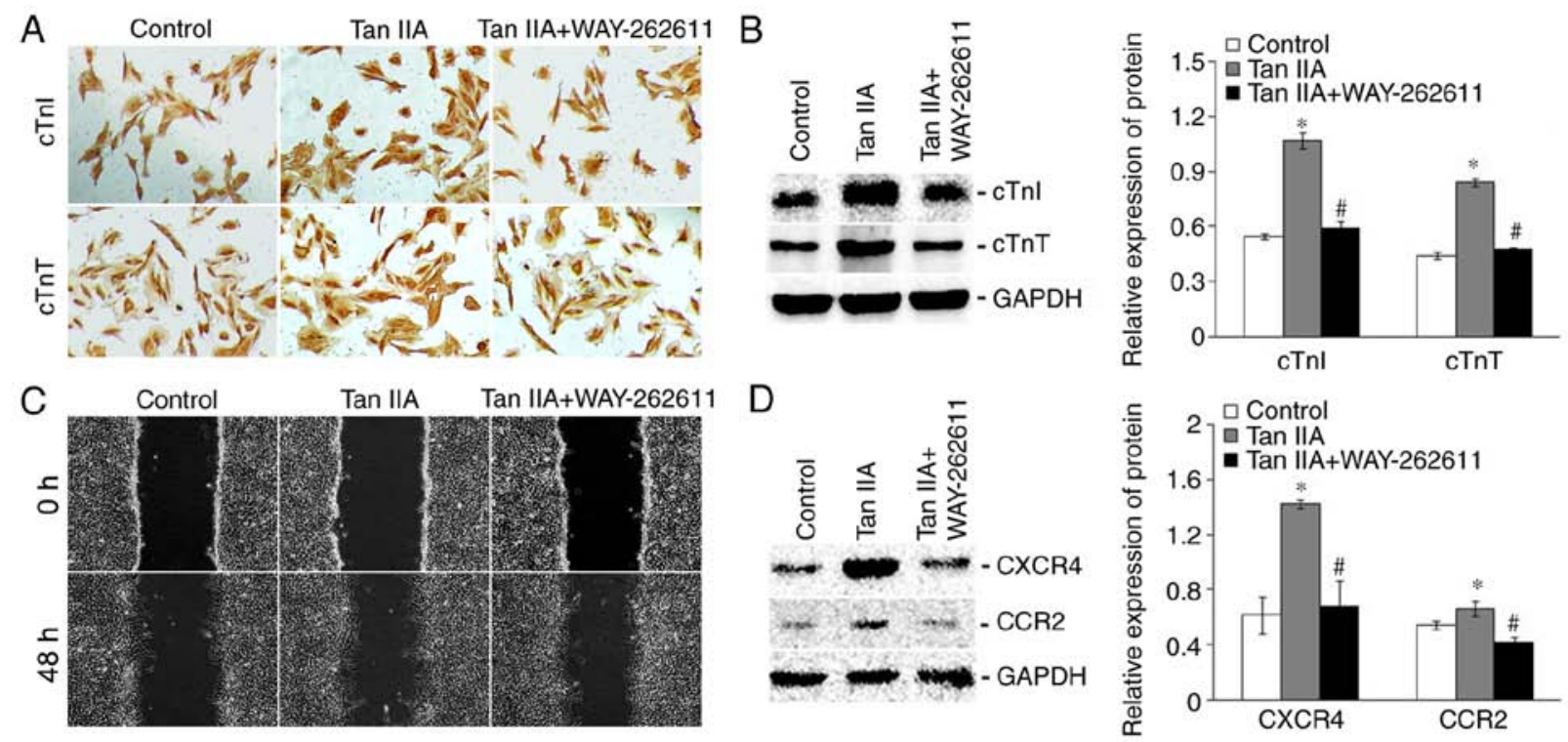

Figure 6. Effects of $\beta$-catenin agonist WAY-262611 on the promoting effect of TanIIA on cardiac differentiation and cell motility. H9c2 cells were treated with TanIIA or TanIIA combined with $\beta$-catenin agonist WAY-262611 for 7 days. At the end of incubation, (A) immunohistochemistry was used to evaluate the expression of cTn (magnification, x200), (B) western blotting was used to further corroborate expression of cTn in the cells, (C) scratch assay was used to evaluate cell motility (magnification, x100) and (D) western blotting was used to assess the expression of chemokine receptors related to cell motility CXCR4 and CCR2. In the immunohistochemical study, cell staining intensity was found to be positively correlated with the expression level of cTn. ${ }^{*} \mathrm{P}<0.05$ vs. the control; ${ }^{~} \mathrm{P}<0.05$ vs. TanIIA alone. TanIIA, Tanshinone II A; cTn, cardiac troponin; CXCR4, C-X-C chemokine receptor type 4; CCR2, C-C chemokine receptor type 2 .

proliferation caused by higher concentration of TanIIA arose from cell apoptosis. In view of the above, $0.6 \mathrm{mg} / 1$ TanIIA was preferred for better assessment of its effect on cardiac differentiation. To further corroborated that the inhibition of cell proliferation may be caused by cell differentiation rather than cell apoptosis, western blotting was then used to evaluate the expression of proteins related to cell cycle (CDK4, CDK6 and cyclin D1), proliferation (c-Myc, Oct4 and PCNA) and apoptosis (cleaved caspase-3). The results demonstrated that TanIIA could inhibit the proliferation of H9c2 cells by downregulating the expression of proteins related to cell cycle (CDK4, CDK6 and cyclin D1) and proliferation (c-Myc, Oct4 and PCNA), while the cell proliferation inhibition was not accompanied by changes in cell apoptosis or the expression of pro-apoptotic proteins caspase- 3 and cleaved caspase- 3 . Cardiac differentiation is a step-by-step process, starting from an early stage, through a middle stage, and finally into the mature stage. At different differentiation stages, the characteristic genes and proteins expressed by differentiated cells also differ $(35,36)$. Among them, cTn is the characteristic protein expressed by the differentiated cells in the middle-late or mature cardiac differentiation stages, and this is also the reason why cTn is used as the standard biomarker for detecting acute myocardial necrosis $(35,36)$. The H9c2 cells used in the present study are still in the middle stage of cardiac differentiation before being induced to differentiate into cardiomyocytes and although they also express cTn, the expression level is significantly lower compared with cells in the late or mature stage of cardiac differentiation. Therefore, after evaluating the effect of TanIIA on the proliferation of $\mathrm{H} 9 \mathrm{c} 2$ cells, the effect of TanIIA on the differentiation of H9c2 into cardiomyocytes was then determined by detecting the expression level of cTn. The results demonstrated that the expression of cTnI and cTnT in the cells treated by TanIIA was significantly higher than that in the cells untreated by TanIIA, which suggested that TanIIA promoted cardiac differentiation of $\mathrm{H} 9 \mathrm{c} 2$ cells. In addition, the results also indicated that TanIIA could significantly improve the motility of the differentiated cells and promote their migration towards the injured area by upregulating the expression of chemokine receptors CXCR4 and CCR2.

The canonical Wnt/ $\beta$-catenin pathway regulates stem cell pluripotency and cell fate decisions during development (18). Various studies indicate that $\mathrm{Wnt} / \beta$-catenin signaling is required for mesoderm formation, while, following mesoderm formation, Wnt/ $\beta$-catenin signaling inhibition can efficiently specify cells to cardiomyocyte fates (19-21). It is known that in the absence of Wnt, $\beta$-catenin is phosphorylated by the synergy of CK1 and APC/Axin/GSK-3 $\beta$ complex, and the phosphorylated $\beta$-catenin is recognized by $\beta$-TrCP, resulting in its ubiquitination and degradation (37). As $\beta$-catenin is degraded, the expression of its downstream targets related to cell proliferation, such as cyclin D1, c-Myc and Oct4, is decreased resulting in cell proliferation inhibition (38). Therefore, upregulating the negative regulators of Wnt/ $\beta$-catenin pathways such as GSK-3 $\beta$, APC or Axin can lead to the degradation of $\beta$-catenin, thereby inhibiting cell proliferation and promoting cardiac differentiation. The results of the present study validated that TanIIA treatment induced a decreased expression of $\beta$-catenin arising from the increased expression of GSK-3 $\beta$ and APC, thus inhibiting cell proliferation and promoting cardiac differentiation. Wnt11 and Wnt5a mainly trigger noncanonical Wnt signaling (19). Wnt11 has been found to be essential for cardiac formation (20), and Wnt5a has also been shown to have cardiac-promoting activity (21). The results of the present study demonstrated 
that TanIIA treatment also induced the increased expression of Wnt11 and Wnt5a, thus promoting cardiac differentiation. In addition, previous studies have also indicated that both Wnt11 and Wnt5a contribute to cardiac development and differentiation by promoting the degradation of $\beta$-catenin that appears to be independent of the cytoplasmic destruction complex $(22,23)$. This suggests that TanIIA promotes cardiac differentiation by inhibiting the canonical signaling pathway through increasing the expression of GSK-3 $\beta$ and APC and simultaneously increasing expression of Wnt11 and Wnt5a. The present study used $\beta$-catenin agonist WAY-262611 to intervene in $\mathrm{H} 9 \mathrm{c} 2$ cells treated with TanIIA. As expected, the results demonstrated that following WAY-262611 intervention, the H9c2 cells treated with TanIIA demonstrated a less clear trend of differentiation into cardiomyocytes and reduced improvement in cell motility.

In conclusion, the data from the present study demonstrated that TanIIA promotes cardiac differentiation and improves cell motility through modulation of the Wnt/ $\beta$-catenin signaling pathway. Following the pharmacological actions of TanIIA and the result above, it is proposed that TanIIA may serve beneficial roles in myocardial regeneration following stem cell transplantation. In addition, the results also indicated the novel idea that the TanIIA may not be safe for pregnant women as it could adversely affect the normal cardiac development process; this hypothesis requires further study.

\section{Acknowledgements}

The authors would like to thank Professor Jianhua Huang (The First Affiliated Hospital of Jinzhou Medical University, Jinzhou, China) for helping with the experimental design and Professor Wei Wang (The First Affiliated Hospital of Jinzhou Medical University, Jinzhou, China) for the technical assistance.

\section{Funding}

The present study was supported by the National Natural Science Foundation (grant no. 81303255) and the Natural Science Foundation of Liaoning Province (grant no. 2019-MS-142).

\section{Availability of data and materials}

All data generated or analyzed during this study are included in this published article.

\section{Authors' contributions}

KL, SL and HL designed the study. XW, CF and CW conceived and performed the experiments. KL and XW performed the statistical analysis and analyzed the data. KL wrote the manuscript. All authors read and approved the final manuscript.

\section{Ethics approval and consent to participate}

Not applicable.

\section{Patient consent for publication}

Not applicable.

\section{Competing interests}

The authors declare that they have no competing interests.

\section{References}

1. Benjamin EJ, Muntner P, Alonso A, Bittencourt MS, Callaway CW, Carson AP, Chamberlain AM, Chang AR, Cheng S, Das SR, et al: Heart disease and stroke statistics-2019 update: A report from the American Heart Association. Circulation 139: e56-e528, 2019.

2. Yu H, Lu K, Zhu J and Wang J: Stem cell therapy for ischemic heart diseases. Br Med Bull 121: 135-154, 2017.

3. Kadota S and Shiba Y: Pluripotent stem cell-derived cardiomyocyte transplantation for heart disease treatment. Curr Cardiol Rep 21: 73, 2019.

4. Fouts K, Fernandes B, Mal N, Liu J and Laurita KR: Electrophysiological consequence of skeletal myoblast transplantation in normal and infarcted canine myocardium. Heart Rhythm 3: 452-461, 2006.

5. Miura K, Okada Y, Aoi T, Okada A, Takahashi K, Okita K, Nakagawa M, Koyanagi M, Tanabe K, Ohnuki M, et al: Variation in the safety of induced pluripotent stem cell lines. Nat Biotechnol 27: 743-745, 2009.

6. Sougawa N, Miyagawa S, Fukushima S, Kawamura A, Yokoyama J, Ito E, Harada A, Okimoto K, Mochizuki-Oda N, Saito A and Sawa Y: Immunologic targeting of CD30 eliminates tumourigenic human pluripotent stem cells, allowing safer clinical application of hiPSC-based cell therapy. Sci Rep 8: 3726, 2018.

7. Gulbins H, Meiser BM, Reichenspurner H and Reichart B: Cell transplantation-a potential therapy for cardiac repair in the future? Heart Surg Forum 5: E28-E34, 2002.

8. Hashimoto $\mathrm{H}$, Olson EN and Bassel-Duby R: Therapeutic approaches for cardiac regeneration and repair. Nat Rev Cardiol 15: 585-600, 2018.

9. Kang YJ, Jin UH, Chang HW, Son JK, Lee SH, Son KH, Chang YC, Lee YC and $\mathrm{Kim} \mathrm{CH}$ : Inhibition of microsomal triglyceride transfer protein expression and atherogenic risk factor apolipoprotein B100 secretion by tanshinone IIA in HepG2 cells. Phytother Res 22: 1640-1645, 2008.

10. Adams JD, Wang R, Yang J and Lien EJ: Preclinical and clinical examinations of Salvia miltiorrhiza and its tanshinones in ischemic conditions. Chin Med 1: 3, 2006.

11. Li CZ, Yang SC and Zhao FD: Effects of tanshinone II-A sulfonate on thrombus formation, platelet and blood coagulation in rats and mice. Zhongguo Yao Li Xue Bao 5: 39-42, 1984 (In Chinese).

12. Jang SI, Kim HJ, Kim YJ, Jeong SI and You YO: Tanshinone IIA inhibits LPS-induced NF-kappaB activation in RAW 264.7 cells: Possible involvement of the NIK-IKK, ERK1/2, p38 and JNK pathways. Eur J Pharmacol 542: 1-7, 2006.

13. Wu GB, Zhou EX and Qing DX: Tanshinone II(A) elicited vasodilation in rat coronary arteriole: Roles of nitric oxide and potassium channels. Eur J Pharmacol 617: 102-107, 2009.

14. Wan AK, Leung SW, Zhu DY and Man RY: Vascular effects of different lipophilic components of 'Danshen', a traditional Chinese medicine, in the isolated porcine coronary artery. J Nat Prod 71: 1825-1828, 2008.

15. Shang Q, Xu H and Huang L: TanshinoneIIA: A promising natural cardioprotective agent. Evid Based Complement Alternat Med 2012: 716459, 2012.

16. Wu TW, Zeng LH, Fung KP, Wu J, Pang H, Grey AA, Weisel RD and Wang JY: Effect of sodium tanshinone IIA sulfonate in the rabbit myocardium and on human cardiomyocytes and vascular endothelial cells. Biochem Pharmacol 46: 2327-2332, 1993.

17. Li K, Song J, Zhao Q, Wang B, Zhang Y, Wang X, Tang T and Li S: Effective component of Salvia miltiorrhiza in promoting cardiomyogenic differentiation of human placenta-derived mesenchymal stem cells. Int J Mol Med 41: 962-968, 2018.

18. Gessert S and Kühl M: The multiple phases and faces of wnt signaling during cardiac differentiation and development. Circ Res 107: 186-199, 2010. 
19. Parikh A, Wu J, Blanton RM and Tzanakakis ES: Signaling pathways and gene regulatory networks in cardiomyocyte differentiation. Tissue Eng Part B Rev 21: 377-392, 2015.

20. Pandur P, Läsche M, Eisenberg LM and Kühl M: Wnt-11 activation of a non-canonical Wnt signalling pathway is required for cardiogenesis. Nature 418: 636-641, 2002.

21. Li D, Sinha T, Ajima R, Seo HS, Yamaguchi TP and Wang J: Spatial regulation of cell cohesion by Wnt5a during second heart field progenitor deployment. Dev Biol 412: 18-31, 2016.

22. Bisson JA, Mills B, Paul Helt JC, Zwaka TP and Cohen ED: Wnt5a and Wnt11 inhibit the canonical Wnt pathway and promote cardiac progenitor development via the Caspase-dependent degradation of AKT. Dev Biol 398: 80-96, 2015.

23. Cohen ED, Miller MF, Wang Z, Moon RT and Morrisey EE: Wnt5a and Wnt11 are essential for second heart field progenitor development. Development 139: 1931-1940, 2012.

24. Liu AR, Liu L, Chen S, Yang Y, Zhao HJ, Liu L, Guo FM, Lu XM and Qiu HB: Activation of canonical wnt pathway promotes differentiation of mouse bone marrow-derived MSCs into type II alveolar epithelial cells, confers resistance to oxidative stress, and promotes their migration to injured lung tissue in vitro. J Cell Physiol 228: 1270-1283, 2013.

25. Sumi D, Abe K and Himeno S: Arsenite retards the cardiac differentiation of rat cardiac myoblast $\mathrm{H} 9 \mathrm{c} 2$ cells. Biochem Biophys Res Commun 436: 175-179, 2013.

26. Branco AF, Pereira SP, Gonzalez S, Gusev O, Rizvanov AA and Oliveira PJ: Gene expression profiling of $\mathrm{H} 9 \mathrm{c} 2$ myoblast differentiation towards a cardiac-like phenotype. PLoS One 10: e0129303,2015.

27. Shafiq M, Lee SH, Jung Y and Kim SH: Strategies for recruitment of stem cells to treat myocardial infarction. Curr Pharm Des 21: 1584-1597, 2015.

28. Shi M, Li J, Liao L, Chen B, Li B, Chen L, Jia H and Zhao RC: Regulation of CXCR4 expression in human mesenchymal stem cells by cytokine treatment: Role in homing efficiency in NOD/SCID mice. Haematologica 92: 897-904, 2007.

29. Andres RH, Choi R, Pendharkar AV, Gaeta X, Wang N, Nathan JK, Chua JY, Lee SW, Palmer TD, Steinberg GK and Guzman R: The CCR2/CCL2 interaction mediates the transendothelial recruitment of intravascularly delivered neural stem cells to the ischemic brain. Stroke 42: 2923-2931, 2011.
30. Carotenuto F, Teodori L, Maccari AM, Delbono L, Orlando G and Di Nardo P: Turning regenerative technologies into treatment to repair myocardial injuries. J Cell Mol Med 24: 2704-2716, 2020.

31. Chen FY, Guo R and Zhang BK: Advances in cardiovascular effects of tanshinone II(A). Zhongguo Zhong Yao Za Zhi 40: 1649-1653, 2015 (In Chinese).

32. Xu W, Yang J and Wu LM: Cardioprotective effects of tanshinone IIA on myocardial ischemia injury in rats. Pharmazie 64: 332-336, 2009

33. Zhang Y, Wei L, Sun D, Cao F, Gao H, Zhao L, Du J, Li Y and Wang H: Tanshinone IIA pretreatment protects myocardium against ischaemia/reperfusion injury through the phosphatidylinositol 3-kinase/Akt-dependent pathway in diabetic rats. Diabetes Obes Metab 12: 316-322, 2010.

34. Li K, Li SZ,Zhang YL and Wang XZ: The effects of dan-shen root on cardiomyogenic differentiation of human placenta-derived mesenchymal stem cells. Biochem Biophys Res Commun 415: 147-151, 2011

35. Hakuno D, Takahashi T, Lammerding J and Lee RT: Focal adhesion kinase signaling regulates cardiogenesis of embryonic stem cells. J Biol Chem 280: 39534-39544, 2005.

36. Wei H, Juhasz O, Li J, Tarasova YS and Boheler KR: Embryonic stem cells and cardiomyocyte differentiation: Phenotypic and molecular analyses. J Cell Mol Med 9: 804-817, 2005.

37. Stamos JL and Weis WI: The $\beta$-catenin destruction complex. Cold Spring Harb Perspect Biol 5: a007898, 2013.

38. Mohammed MK, Shao C, Wang J, Wei Q, Wang X, Collier Z, Tang S, Liu H, Zhang F, Huang J, et al: Wnt/ $\beta$-catenin signaling plays an ever-expanding role in stem cell self-renewal, tumorigenesis and cancer chemoresistance. Genes Dis 3: 11-40, 2016.

This work is licensed under a Creative Commons Attribution-NonCommercial-NoDerivatives 4.0 International (CC BY-NC-ND 4.0) License. 